\title{
THE MALLIAVIN DERIVATIVE FOR G ENERALIZATION OF FRACTIONAL BESSEL TYPE PROCESS
}

\author{
Vu Thi Huong
}

University of Transport and Communications

\begin{tabular}{|c|c|}
\hline ARTICLE INFO & \multirow{9}{*}{$\begin{array}{l}\text { This paper considersa generalization of fractional Bessel type process. } \\
\text { It is also a type of singular stochastic differential equations driven by } \\
\text { fractional Brownian motion which were studied by some authors. } \\
\text { Undersome assumptions of coefficients, this equation has a unique } \\
\text { positive solution. The main purpose of this paper is to show the } \\
\text { formula of the Malliavin derivative for this process. The techniques } \\
\text { of Malliavin calculus were applied for stochastic differential } \\
\text { equations driven by a fractional Brownian motion. We obtain that the } \\
\text { Malliavin derivative for this process is an exponent function of the } \\
\text { drift coefficient's derivative. This result is useful to estimate inverse } \\
\text { moments of the solution. From that, we can estimate the rate of } \\
\text { convegence of the numerical approximation in the } \mathrm{L}_{\mathrm{p}} \text { - norm. }\end{array}$} \\
\hline Received: 07/4/2021 & \\
\hline Revised: $28 / 5 / 2021$ & \\
\hline Published: 31/5/2021 & \\
\hline KEYWORDS & \\
\hline Fractional Brownian motion & \\
\hline Fractional Bessel process & \\
\hline $\begin{array}{l}\text { Fractional stochastic differential } \\
\text { equation }\end{array}$ & \\
\hline & \\
\hline
\end{tabular}

Malliavin calculus

\section{ĐẠO HÀM MALLIAVIN CỦA QUÁ TRÌNH BESSEL PHÂN THỨ DẠNG TỔNG QUÁT}

Vũ Thị Hương

Truờng Đại học Giao thông Vận tải

\begin{tabular}{|c|c|}
\hline THÔNG TIN BÀI BÁO & TÓM TẮT \\
\hline Ngày nhận bài: 07/4/2021 & \multirow{8}{*}{ 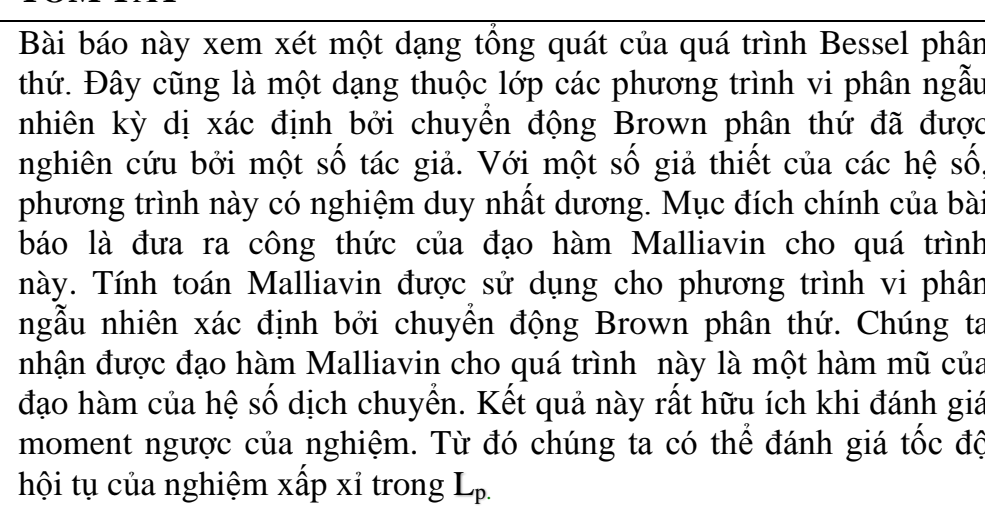 } \\
\hline Ngày hoàn thiện: $28 / 5 / 2021$ & \\
\hline Ngày đăng: 31/5/2021 & \\
\hline TÙ KHÓA & \\
\hline Chuyển động Brown phân thứ & \\
\hline Quá trình Bessel phân thứ & \\
\hline $\begin{array}{l}\text { Phương trình vi phân ngẫu nhiên } \\
\text { phân thứ }\end{array}$ & \\
\hline & \\
\hline
\end{tabular}

Tính toán Malliavin

DOI: $\underline{\text { https://doi.org/10.34238/tnu-jst.4243 }}$

Email:vthuong@utc.edu.vn 


\section{Introduction}

In [1], the authors considered the following singular stochastic differential equation driven by fractional Brownian motion with Hurst parameter $H>1 / 2$.

$$
X_{t}=x_{0}+\int_{0}^{t} \frac{K}{X_{s}} d s+B_{t}^{H}
$$

where $K>0$ is a constant. They showed the formula for the Malliavin derivative of the solution and then estimated negative moments. In [2], Mishura and Yurchenko-Tytarenko studied the fractional stochastic differential equation

$$
d Y(t)=\frac{1}{2}\left(\frac{k}{Y(t)}-a Y(t)\right) d t+\frac{1}{2} \sigma d B_{t}^{H}, \quad Y(0)>0, k>0,
$$

They considered the probability of hitting zero of the solution. This equation was also studied in [3]. Authors introduced an implicit Euler scheme and showed that the scheme strongly converges at the rate of order 1 . They also gave the boundedness of inverse moments of the numerical solution and derived the Malliavin derivative of the numerical solution. In this paper, we consider a more general singular stochastic differential equation driven by fractional Brownian motion. More precisely, we study a generalization of Bessel type process $Y=(Y(t))_{0 \leq t \leq T}$ satisfying the following SDEs,

$$
d Y(t)=\left(\frac{k}{Y(t)}+b(t, Y(t))\right) d t+\sigma d B^{H}(t)
$$

where $0 \leq t \leq T, Y(0)>0$ and $B^{H}$ is a fractional Brownian motion with the Hurst parameter $H>\frac{1}{2}$ defined in a complete probability space $(\Omega, \mathcal{F}, \mathbb{P})$ with a filtration $\left\{\mathcal{F}_{t}, t \in[0, T]\right\}$ satisfying the usual condition. Fix $T>0$ and we consider equation (3) on the interval $[0, T]$. We suppose that $k>0$ and the coefficient $b=b(t, x):[0,+\infty) \times \mathbb{R} \rightarrow \mathbb{R}$ is a mesurable function and globally Lipschitz continuous with respect to $x$, linearly growth with respect to $x$. It means that there exists positive constants $L, C$ such that the following conditions hold:

$\left.A_{1}\right)|b(t, x)-b(t, y)| \leq L|x-y|$, for all $x, y \in \mathbb{R}$ and $t \in[0, T] ;$

$\left.A_{2}\right)|b(t, x)| \leq C(1+|x|)$, for all $x \in \mathbb{R}$ and $t \in[0, T]$;

$\left.A_{3}\right) b(t, x)$ has a continuous partial derivative with respect to $x$.

The existence and uniqueness of solution for generalization of the fractional Bessel type process was proved in [4] by the following Theorems

Theorem 1.1. Assume that conditions $\left(A_{1}\right)-\left(A_{2}\right)$ are satisfied. Then for each $T>0$ equation (3) has a unique solution on $[0, T]$. 
Lemma 1.2. Let $(Y(t))_{0 \leq t \leq T}$ be the solution of equation (3) then $Y(t)>0$ for all $t \in[0, T]$ almost surely.

The main purpose of this paper is to study the Malliavin derivative of the solution using Malliavin calculus for stochastic differential equations driven by a fractional Brownian motion. So, firstly, we recall some basic facts on Malliavin calculus (see [5], [6], [7] ).

\section{Malliavin calculus}

Fix a time interval $[0, T]$. We consider a fractional Brownian motion $\left\{B^{H}(t)\right\}_{t \in[0, T]}$. We note that $E\left(B^{H}(s) \cdot B^{H}(t)\right)=R_{H}(s, t)$ where

$$
R_{H}(s, t)=\frac{1}{2}\left(t^{2 H}+s^{2 H}-|t-s|^{2 H}\right) .
$$

We denote by $\mathcal{E}$ the set of real valued step functions on $[0, T]$. Let $\mathcal{H}$ be the Hibert space defined as the closure of $\mathcal{E}$ with respect to the scalar product

$$
\left\langle 1_{[0, t]}, 1_{[0, s]}\right\rangle_{\mathcal{H}}=R_{H}(t, s) .
$$

On the other hand, in [6], authors showed that the covariance $R_{H}(t, s)$ can be written as

$$
R_{H}(t, s)=\alpha_{H} \int_{0}^{t} \int_{0}^{s}|r-u|^{2 H-2} d u d r=\int_{0}^{t \wedge s} K_{H}(t, r) K_{H}(s, r) d r
$$

where $\alpha_{H}=H(2 H-1), K_{H}(t, s)$ is the square integrable kernel defined by

$$
K_{H}(t, s)=c_{H} s^{\frac{1}{2}-H} \int_{0}^{t}(u-s)^{H-\frac{3}{2}} u^{H-\frac{1}{2}} d u,
$$

for $t>s$, where $c_{H}=\sqrt{\frac{H(2 H-1)}{\beta\left(2-2 H, H-\frac{1}{2)}\right.}}$ and $\beta$ denote the Beta function. We put $K_{H}(t, s)=0$ if $t \leq s$.

It implies that for any pair of step functions $\varphi, \psi \in \mathcal{E}$ we have

$$
\langle\varphi, \psi\rangle=\alpha_{H} \int_{0}^{T} \int_{0}^{T}|r-u|^{2 H-2} \varphi_{r} \psi_{u} d u d r
$$

The mapping $1_{[0, t]} \mapsto B^{H}(t)$ can be extend to an isometry between $\mathcal{H}$ and the Gaussian space associated with $B^{H}$. Denote this isometry by $\varphi \mapsto B^{H}(\varphi)$.

Definition 2.1. Let $\mathcal{S}$ be the space of smooth and cylindrical random variables of the form

$$
F=f\left(B^{H}\left(\varphi_{1}\right), \ldots, B^{H}\left(\varphi_{n}\right)\right)
$$


where $n \geq 1, f \in C_{b}^{\infty}\left(\mathbb{R}^{n}\right)$. We define the derivative operator $D F$ on $F \in \mathcal{S}$ as the $\mathcal{H}$-valued random variable

$$
D F=\sum_{i=1}^{n} \frac{\partial F}{\partial x_{i}}\left(B^{H}\left(\varphi_{1}\right), \ldots, B^{H}\left(\varphi_{n}\right)\right) \varphi_{i} .
$$

We call the derivative operator DF as the Malliavin derivative of random variable $F$.

We consider the linear operator $K_{H}^{*}: \mathcal{E} \rightarrow L^{2}([0, T])$ defined by

$$
\left(K_{H}^{*} \varphi\right)(s)=\int_{s}^{T} \varphi(t) \frac{\partial K_{H}}{\partial t}(t, s) d t .
$$

Notice that $\left(K_{H}^{*}\left(1_{[0, t]}\right)(s)=K_{H}(t, s) 1_{[0, t]}(s)\right.$. Then for any $\varphi, \phi \in \mathcal{E}$ we have

$$
\langle\varphi, \phi\rangle_{\mathcal{H}}=\left\langle K_{H}^{*} \varphi, K_{H}^{*} \phi\right\rangle_{L^{2}([0, T])}=E\left(B^{H}(\varphi) B^{H}(\phi)\right)
$$

Then $K_{H}^{*}$ provides an isometry between the Hilbert space $\mathcal{H}$ and a closed subspace of $L^{2}([0, T])$.

We define the operator $\mathcal{K}_{H}: L^{2}([0, T]) \rightarrow \mathcal{H}_{H}=\mathcal{K}_{H}\left(L^{2}([0, T])\right.$

$$
\left(\mathcal{K}_{H} h\right)(t)=\int_{0}^{t} K_{H}(t, s) h(s) d s .
$$

We denote by $\mathcal{R}_{H}=\mathcal{K}_{H} \circ K_{H}^{*}: \mathcal{H} \rightarrow \mathcal{H}_{H}$ the operator defined as following

$$
\mathcal{R}_{H} \varphi=\int_{0}^{\cdot} K_{H}(., s)\left(K_{H}^{*} \varphi\right)(s) d s .
$$

In [7], authors proved that for any $\varphi \in \mathcal{H}, \mathcal{R}_{H} \varphi$ is Holder continuous of order $H$.

The following Lemma is an integration by parts formula (see Lemma 11 in [7])

Lemma 2.2. Let $\lambda$ such that $\lambda+H>1, f \in C^{\lambda}(0, T)$ and $h \in \mathcal{H}$. Then it holds that

$$
\int_{0}^{T} f(r) d\left(\mathcal{R}_{H} \varphi\right)(r)=\int_{0}^{T} f(r)\left(\int_{0}^{r} \frac{\partial K_{H}}{\partial r}(r, t)\left(K_{H}^{*} \varphi(t) d t\right) d r .\right.
$$

Let $(Y(t))_{t \in[0, T]}$ be the solution of equation (3).

Definition 2.3. The derivative defined by

$$
D_{\mathcal{R}_{H} \varphi} Y(t)=\left.\frac{d}{d \epsilon} Y(t)\left(\omega+\epsilon \mathcal{R}_{H} \varphi\right)\right|_{\epsilon=0},
$$

exists for all $\varphi \in \mathcal{H}$ (see [7]).

Lemma 2.4. The derivative $D_{\mathcal{R}_{H} \varphi} Y(t)$ coincides with $\langle D Y(t), \varphi\rangle_{\mathcal{H}}$, where $D Y$ is the Malliavin derivative of $Y$ (see [7]). 


\section{Estimate the Malliavin derivative of solution}

The main result of this paper provides an estimation for the Malliavin derivative of solution $Y(t)$ of equation (3) which is stated as the following Theorem

Theorem 3.1. Assume that conditions $\left(A_{1}\right)-\left(A_{3}\right)$ are satisfied and $Y(t)$ is the solution of equation (3), then for any $t>0$, we have

$$
D Y(t)=\sigma \cdot \exp \left(\int^{t}\left[\frac{k}{Y^{2}(r)}+\frac{\partial b}{\partial y}(r, Y(r))\right] d r\right) \mathbf{1}_{[0, t]} .
$$

Proof. Fix a time interval $[0, T]$. Firstly, we compute $\langle D Y(t), \varphi\rangle_{\mathcal{H}}, \varphi \in \mathcal{H}$. Setting $h=$ $\mathcal{R}_{H} \varphi$. By equation (14) we have

$$
\langle D Y(t), \varphi\rangle \mathcal{H}=D_{\mathcal{R}_{H} \varphi} Y(t)=\left.\frac{d Y^{\epsilon}(t)}{d \epsilon}\right|_{\epsilon}=0,
$$

where $Y^{\epsilon}(t)$ is the solution of the equation

$$
Y^{\epsilon}(t)=Y(0)+\int_{0}^{t}\left[\frac{k}{Y^{\epsilon}(s)}+b\left(s, Y^{\epsilon}(s)\right)\right] d s+\sigma B^{H}(t)+\epsilon . \sigma h(t), t \in[0, T], \epsilon \in[0,1] .
$$

It leads to

$$
Y^{\epsilon}(t)-Y(t)=\int_{0}^{t}\left[\frac{k}{Y^{\epsilon}(s)}+b\left(s, Y^{\epsilon}(s)\right)-\left(\frac{k}{Y(s)}+b(s, Y(s))\right)\right] d s+\epsilon . \sigma h(t) .
$$

From assumption $A_{3}$, using Taylor expansion, we have

$$
Y^{\epsilon}(t)-Y(t)=\int_{0}^{t} \partial_{y} f\left(s, Y(s)+\theta_{s}^{\epsilon}\left(Y^{\epsilon}(s)-Y(s)\right)\right)\left(Y^{\epsilon}(s)-Y(s)\right) d s+\epsilon . \sigma h(t)
$$

where $f=f(t, y)=\frac{k}{y}+b(t, y), \theta_{s}^{\epsilon}$ between 0 and 1 . This equation is a first order linear partial differential equation. Then the formula of the solution of equation is that

$$
Y^{\epsilon}(t)-Y(t)=\epsilon \cdot \sigma \int_{0}^{t} \exp \left(\int_{s}^{t} \partial_{y} f\left(r, Y(r)+\theta_{r}^{\epsilon}\left(Y^{\epsilon}(r)-Y(r)\right)\right) d r\right) d h(s) .
$$

Using Lemma 2.2, we have

$$
\begin{aligned}
Y^{\epsilon}(t)-Y(t) & =\epsilon \cdot \sigma \int_{0}^{t} \exp \left(\int_{s}^{t} \partial_{y} f\left(r, Y(r)+\theta_{r}^{\epsilon}\left(Y^{\epsilon}(r)-Y(r)\right)\right) d r\right)\left(\int_{0}^{s} \frac{\partial K_{H}}{\partial s}(s, u)\left(K_{H}^{*} \varphi\right)(u) d u\right) d s \\
& =\epsilon \cdot \sigma \int_{0}^{t}\left(\int_{u}^{t} \exp \left(\int_{s}^{t} \partial_{y} f\left(r, Y(r)+\theta_{r}^{\epsilon}\left(Y^{\epsilon}(r)-Y(r)\right)\right) d r\right) \frac{\partial K_{H}}{\partial s}(s, u) d s\right)\left(K_{H}^{*} \varphi\right)(u) d u
\end{aligned}
$$


By the definition of the operator $K_{H}^{*}$ we have

$$
\begin{aligned}
Y^{\epsilon}(t)-Y(t) & =\epsilon \cdot \sigma \int_{0}^{t}\left(K_{H}^{*} \varphi\right)(u)\left(K_{H}^{*}\left(\exp \left(\int^{t} \partial_{y} f\left(r, Y(r)+\theta_{r}^{\epsilon}\left(Y^{\epsilon}(r)-Y(r)\right)\right) d r\right)\right)\right)(u) d u \\
& =\epsilon \cdot \sigma\left\langle\varphi, \exp \left(\int^{t} \partial_{y} f\left(r, Y(r)+\theta_{r}^{\epsilon}\left(Y^{\epsilon}(r)-Y(r)\right)\right) d r\right)\right\rangle_{\mathcal{H}} \\
& =\alpha_{H} \epsilon \cdot \sigma \int_{0}^{t} \int_{0}^{t} \varphi(s) \exp \left(\int_{u}^{t} \partial_{y} f\left(r, Y(r)+\theta_{r}^{\epsilon}\left(Y^{\epsilon}(r)-Y(r)\right)\right) d r\right)|s-u|^{2 H-2} d u d s .
\end{aligned}
$$

By assumption that $k>0$ we have

$$
\partial_{y} f(t, y)=\frac{-k}{y^{2}}+\partial_{y} b(t, y) \leq \partial y b(t, y)
$$

But from assumption $A_{1}$,

$$
\frac{b(t, x)-b(t, y)}{x-y} \leq \frac{|b(t, x)-b(t, y)|}{|x-y|} \leq L .
$$

Then $\partial_{y} b(t, y) \leq L$. It mean that $\partial_{y} f(t, y)$ is bounded. As a consequence,

$$
\begin{aligned}
\lim \frac{Y^{\epsilon}(t)-Y(t)}{\epsilon}= & \alpha_{H} \cdot \sigma \int_{0}^{t} \int_{0}^{t} \varphi(s) \exp \left(\int_{u}^{t} \partial_{y} f(r, Y(r) d r)|s-u|^{2 H-2} d u d s\right. \\
& =\sigma\left\langle\varphi, \exp \left(\int^{t} \partial_{y} f(r, Y(r) d r) \mathbf{1}_{[0, t]}\right\rangle_{\mathcal{H}},\right.
\end{aligned}
$$

where the limit holds almost surely and in $L^{2}(\Omega)$.

Together with equation (16), it leads to

$$
\begin{aligned}
D Y(t) & =\sigma \cdot \exp \left(\int^{t} \partial_{y} f(r, Y(r) d r) \mathbf{1}_{[0, t]}\right. \\
& =\sigma \cdot \exp \left(\int_{.}^{t}\left[\frac{k}{Y^{2}(r)}+\frac{\partial b}{\partial y}(r, Y(r))\right] d r\right) \mathbf{1}_{[0, t]} .
\end{aligned}
$$

We obtain the formula of the Malliavin derivative $D Y$.

\section{Conclusion}

The main result of this paper is to prove the formula of the Malliavin derivative for a type of singular process driven fractional Brownian motion. From this formula, in the future, we can estimate inverse moments of this process which is necessary in showing the rate convegence of the numerical approximation in the $L_{p}$ - norm. 


\section{REFERENCES}

[1] Y. Hu, D. Nualart and X. Song, "A singular stochastic differential equation driven by fractional Brownian motion," Statistics Probability Letters, vol. 78, no. 14, pp. 2075 2085, 2008.

[2] Y. Mishura, Anton Yurchenko-Tytarenko, "Fractional Cox-Ingersoll-Ross process with non-zero "mean"," Mod. Stoch. Theory Appl., vol. 5, no. 1, pp. 99 - 111, 2018.

[3] J. Hong, C. Huang, M. Kamrani, X.Wang, " Optimal strong convergence rate of a backward Euler type scheme for the Cox-Ingersoll-Ross model driven by fractional Brownian motion", Stochastic Processes and their Applications, vol.130, no. 5, pp. 26752692, 2020.

[4] T. H. Vu, "Existence and uniqueness of solution for generalization of fractional Bessel type process," (in Vietnamese), TNU Journal of Science and Technology, vol. 225, no. 02: Natural Sciences - Engineering - Technology, pp. 39-44, 2020.

[5] F. Biagini, Y. Hu, B. Oksendal and T. Zhang, Stochastic Calculus for Fractional Brownian Motion and Applications, Springer, London, 2008.

[6] D. Nualart, The Malliavin Calculus and Related Topics, 2nd Edition, SpringerVerlag Berlin Heidelberg, 2006.

[7] D. Nualart and B. Saussereau, "Malliavin calculus for stochastic differential equations driven by a fractional Brownian motion," Stochastic processes and their applications, vol. 119, no. 2, pp. 391-409, February 2009. 